\title{
Network Size Estimation in VANETs
}

\author{
Yaser Khamayseh \\ Computer Science Dept, Jordan University of Science and Technology, Irbid-Jordan \\ Tel: +962-779060324 E-mail: yaser@just.edu.jo
}

Muneer BaniYassein

Computer Science Dept, Jordan University of Science and Technology, Irbid-Jordan

Tel: +962-796166131 $\quad$ E-mail: masadeh@just.edu.jo

Mariam AbdAlghani

Computer Science Dept, Jordan University of Science and Technology, Irbid-Jordan

Tel: +962-772212975_E-mail: mariam_bataineh@yahoo.com

Constandinos X. Mavromoustakis

Department of Computer Science, University of Nicosia, Nicosia, Cyprus

Tel: +357-22841500Ｅ-mail: mavromoustakis.c@unic.ac.cy

Received: June, 10, 2013 Accepted: October 25, $2013 \quad$ Published: October 31, 2013

DOI: $10.5296 / n p a . v 5 i 3.3838$

URL: http://dx.doi.org/10.5296/npa.v5i3.3838

\begin{abstract}
Vehicular Ad-Hoc Networks (VANETs) is a branch of Ad-Hoc Networks that covers wide area and nodes are typically moving vehicles. The power level available in the vehicles is relatively higher than the power level available in other types of ad hoc networks. The reliability is an important issue in VANETs that is determined by specifying a mobility model and routing protocol. A key concern in designing reliable routing protocols is the network size assumption. Most protocols assume prior knowledge of the network size; however, this assumption requires a careful consideration and needs to be further investigated. Moreover, the neighborhood relationship between nodes is not well defined and estimated. The moving pattern for users is a critical factor in defining this relation. In this Paper, we investigate the influence of mobility models on the network size and neighborhood relationship, and then propose heuristic and distributed algorithm to define the nodes' neighbors and to estimate the network size. The performance of the proposed algorithm is evaluated using simulation.
\end{abstract}

Keywords: VANET, Network Size, Estimation, Distributed. 


\section{Introduction}

In recent years, there has been a great demand on wireless networks and on applications that rely on wireless networks to support users' mobility. To meet such demands, a wireless technology such as ad-hoc networks, has emerged. In ad-hoc networks, no central point exists to monitor and control the networks. Moreover, ad-hoc networks require no infrastructure to operate the network. Examples of emerging ad-hoc networks are Mobile AdHoc Networks (MANETs), Wireless Sensor Networks (WSNs), Peer-to-Peer (P2P) and Vehicle-to Vehicle (V2V). The nodes need to cooperate, act as a router and as a client at the same time, and exchange and share network information to operate the network.

Vehicle Ad-Hoc networks (VANETs) are special type of MANETs, where the vehicles (sensors) are distributed in the roads, moving with high speed, and with large energy resources. VANETs cover wide geographical area and nodes usually move in reserved roads with high speed. The unlimited battery power and storage provided for VANET nodes are a key advantage over the sensor networks that suffer from energy limitations, thus, the network lifetime is longer and it can store as many data as needed. Another motivation of using VANETs is the improved safety and comfort of passengers inside vehicles [1]. And to maintain the liability of vehicles when accidents occur and reduce the traffic congestion [2]. However, VANETs face several challenges; an important challenge for VANETs is the use of radio wave such that anyone can listen to the messages which compromise the security and privacy of the node (vehicle). Moreover, the network may incur relatively high percentage of packet loss due to interference, bad channels conditions, congestion, and insufficient communication range to receive the packets.

The increased number of vehicles on roads in recent years; increases the burden on the administrations to design and provide safer roads. Therefore, challenges on reliability increases. There are several factors that affect the reliability of vehicles on a road, such as: road type, day time, weather, density of the traffic, and the drivers themselves $[3,6]$. We note that it is possible that not all vehicles can join the VANET, as there are some vehicles are not equipped with the necessary hardware. Moreover, routing is very challenging aspect in VANET since the network topology is affected greatly by nodes mobility due to the frequent changes in the network topology [3]. Reliability is a crucial requirement for inter-vehicle communication in order to improve safety and comfort in roads. Thus, an important aspect that requires special attention is reliability of routing protocols in VANETs. A key concern in designing reliable routing protocols is the network size assumption, to our knowledge; researches conducted on routing in VANETs assume prior knowledge of the network size. This paper aims to estimate the network size (i.e., number of nodes in the network).

The unlimited battery power and storage provided for VANETs nodes paved the way for more applications than other networks. VANETs are used to improve the safety and comfort of passengers inside the vehicles [1], maintain the liability of the vehicles when accidents occur, and reduce the traffic congestion [2]. It is estimated that the police vehicles, fire vehicles and Ambulances will associate with this technology for safety purposes. Vehicles are used to collect data from the population and from vehicles' movement (e.g., position, direction, speed, and digital mapping of roads) to create the knowledge about distance between two vehicles and between the vehicles and infrastructure to avoid accidents and traffic jams. There are two types of feedback loops in VANETS; the first one shows some of the interesting and attracting features, such as free parking spaces. The other one provides information to protect the vehicles, such as traffic jams [38]. 
This paper aims at estimating the network size using the node's knowledge and with minimal exchange of information in the network to minimize the network's overhead. The proposed algorithms depend on information that collected from neighbor nodes using hello messages to minimize the network's overhead. The rest of this paper is organized as follows. Section 2 presents some of the related work about VANETS. Section 3 presents the proposed estimation mechanism. Section 4 presents and discusses the obtained simulation results. And, finally, Section 5 concludes the paper and provides some future research directions.

\section{Literature Review}

In the literature, there are many proposed schemes conducted on VANETs varying in their goals and mechanism and they assume the number of nodes is known in advance rather than calculating the network size. They have been classified into different categories: routing in MANETs [22, 23], routing in VANETs [11], comparison between routing in VANETs and in MANETs, feedback loops in VANETs, the scalability in VANETs, Other works focus on mobility models and scenarios of VANETs $[1,4,5]$. For example the work in [39] argues that the number of nodes in an overlay varies depending on the time of the day, hence, knowing the number of nodes in peer-to-peer network is immense challenge for many reasons; the decentralization of systems, and the probability of nodes to fail. The authors in [39] used gossip-based aggregation algorithm for the estimation.

\subsection{Routing in VANETS}

A new routing approach is presented in [11] for highway/freeway VANETs which consists of a unicast destination exploration process, strong forward node selection mechanism and a positional hello mechanism. The work in [12] proposed an inter-vehicle adhoc routing protocol called GyTAR which consist of two types: dynamic selection of the intersections through which a packet must pass to reach its destination and to improve the greedy strategy that used to forward packets between two junctions. The authors in [37] show a survey of five categories of the routing protocols in VANETs: Position based, Topology based, Geo cast, cluster based and broadcast based. Results obtained in [37] show that VANET routing protocols performance depends on various parameters such as; mobility model, and driving environment.

In [33], the authors evaluated the performance of two on-demand routing protocols, DSR and AODV for different factors such as pause time, speed, traffic scenarios and node density which expand from 30 to 150 nodes. The authors in [36] show the difficulties of designing an efficient routing protocol for VANETs as it require packet transmission reliability which is hard to achieve due to frequent disconnection and rapid topology changes in VANETs. The authors combined AODV with Ant Colony Optimization (ACO) algorithm to improve the performance.

Routing protocols in VANETs can be categorized into two types [6]: Topology based routing which uses the information about the network links, example of this type are FSR [16], AODV [17], TORA [18], and DSR [19]. And, Position based routing which uses the information about the location of neighbors, examples of this type are GPSR [20], and GRANT [21]. The authors in [24] compare between routing protocols used in VANET and MANET, [24] concluded that routing protocols used in MANET are not suitable for VANET because it does not give the optimal throughput needed for fast mobility of VANET and because the nodes are moving on reserved roads. The authors in [24] present several protocols that are considered suitable and optimal for both MANET and VANET. And the 
authors in [26] argued that the routing protocols in MANET are different than routing protocol in VANET because the traffic, mobility and network topology are different in VANET than in MANET. The dense traffic in VANET is diverse depending on the time of the day. So they proposed the Distributed Vehicular Broadcast (DV-CAST) protocol which combines the dense, sparse and regular traffics in one broadcast routing.

The research in [31] presents road-based routing protocol which yields better performance than existing routing protocols in city-based environments. This vehicular traffic $(\mathrm{RBVT})$ protocol uses real-time traffic information of vehicles to create road-based paths that built from chain of intersections which are connected to each other in the network. They considered and executed a proactive protocol (RBVT-P) and a reactive protocol (RBVT-R). Simulation results in urban settings show that RBVT-R give better performance in terms of average delivery ratio compared with other existing protocols. And RBVT-P gives better performance in terms of average delay.

Other schemes employ GPS systems to enhance their performance. The work in [29] analyzed and studied the localization requirements for the main applications of VANETs. They analyzed others localization techniques in VANETs to avoid GPS problems, because it is not suitable for some applications and not available all the time. [29] used other techniques such as; Cellular Localization, Dead Reckoning, and Video/Image Localization, which used data fusion techniques to find the correct position of vehicles in network without needing the GPS. The feedback loops in VANET was discussed in [38] such that; the traffic information of street are sent to vehicles and drivers (e.g. the conditions of traffic and the availability of parking space). They introduced two types of feedback loops in specific mobility models (e.g., the Constrained Random Trip model, the Random Trip model and the Manhattan Mobility model).In [32], the authors proposed a managing model for flowing data which used to alert or inform the driver based on Genetic Algorithm (GA).

The authors in [25] utilize the information of vehicles' movement details (e.g., position, direction, speed, and digital mapping of roads) to predict a potential link-breakage event before occurrence. They extract the performance of their proposed simulated scheme, in terms of increasing the access duration of link; decreasing the number of link-breakage events and increasing throughput. In [7], the authors used the flooding approach to handle the broadcast message. Moreover some researchers as in [8] studied the storage capability of vehicle mesh where the local transient information could be saved within a given region for a period of time, without any infrastructure. On the other hand, a new simple algorithms provided in [13] for the expected number of vehicles covered and the probability distribution of proper distance between two vehicles by computing the connectivity and the neighborhood between them.

\subsection{Mobility Models and Scenarios of VANETs}

Mobility models and scenarios are the most important in characterizing what differentiates between MANETs and VANETs. The researchers in [8] use the highway and city environment mobility model and compared the performance against random way point mobility model. The authors in [10] considered the optimality of the next-hop selection between two vehicles on a highway scenario depending on its speed and inter-node distances. And [27] used the VANET scenarios to test the performance of ad hoc routing protocols, such as Dynamic MANET On Demand (DYMO).

New mechanisms of broadcasting studied in [28]; the Directed Route Node Selection (DRNS) and Secure Ring Broadcasting (SRB), where they tried to decrease the 
number of messages broadcasted and to increase the stability of routes. Thus they used two scenarios; city scenarios in high density hours which used for simulating SRB, and highway scenarios which used for DRNS. These categories of routing protocol are used in three different network architectures [6]: Pure cellular/WLAN, Pure ad hoc, and hybrid. There are many mobility models that represent the mobility of the vehicles, and each one has different characteristics [1], here we present some of the models used in the literature: Random walk model, random direction model, random waypoint model, reference point group model, city section model, and freeway model. Moreover, there are many factors that affect the mobility of vehicles in the networks and the network topology [4]: Street layout, block size, average speed, and density of the area. The authors in [14] divide the mobility model into two categories: Macro-mobility and micro-mobility. [15] developed a tool, called MOVE, which generates mobility models for VANET simulations. In MOVE, the road map generation can be done manually, automatically or copied from a real world map. Such as: grid, spider, TIGER and random networks.

The authors in [30] showed how the selfless communication method helps the scalability to be realized which is done to solve the operability challenge in sparse and dense networks. Because greedy nodes give wrong information, it may affect the network reliability. And in [35], the authors take into account the reliability during the join of multicast members by proposing QoS based reliable method (QBRM) that is based on the Fuzzy ARTMAP algorithm to find reliable multicast routing. Simulation results shows that the QoS-based Reliable Multicast Routing performance is improved in terms of the cost and the number of messages that queried while the average of multicast group size was big and the alignment of the rate of joining was near to one, but it achieved poor performance for small groups. The authors in [25] show a strong relationship between repeated path disconnection and vehicles mobility, this finding affect the design of scalable routing algorithms.

\section{Proposed Scheme}

Vehicle Ad-Hoc Networks (VANETs) is a branch of Ad-Hoc Networks that covers wide area and nodes are typically moving vehicles. Now a day, VANETs aims to improve the safety and comfort of passengers inside vehicles, and to maintain the liability of vehicles when accidents occur and reduce the traffic congestion. Networks are connected to each other to form one huge network to facilitate the communication between all vehicles. Key concern in designing reliable routing protocols is the network size assumption, however, to our knowledge, work on routing in VANETs assume prior knowledge of the network size, and the network size estimation for VANETs was not considered in the literature. This section presents the proposed scheme. The proposed scheme consists of four modules; Neighbors Estimation Module, Initialization Module, Estimation of $N_{i}$ and $N$ Module and Update the $S F_{i}$ Module.

\subsection{Neighbors Estimation Module}

The first Module includes an algorithm to estimate the number of neighbors $\left(N_{j}\right)$ for node $j$. Moreover, it estimates the Neighborhood Level $(N L)$, it works by scanning all neighbors of node $j$ using hello message. The module is proposed in Figure 1.

Input:

node $i$, $j$, time $t$, Increment percentage $(I P)$, Decrement Percentage $(D P)$, Start Percentage $(S P)$, Neighbor Level $(N L)$. 
Output:

The neighbors of node $j\left(N_{j}\right)$ and Neighbor Level $(N L)$.

Algorithm:

For each node $i$ that receive hello message

If node $i$ is already neighbor for node $j$

Then Increase $N L$ for $i$ by IP

Else add $i$ to the table and set $N L$ to $S P$

Decrease $N L$ for $i$ by $D P$

Return neighbors of node $j$

Figure 1. Neighbors Estimation Module

Each node $j$ contains a table, called neighbors table, which contains a list of node's $j$ neighbors that are within its transmission range. Once a node is inserted to the list (i.e. new neighbor for node $j$ ), it is assigned an initial value called Start Percentage $(S P)$, on the other hand, for existing nodes in the table (i.e. old neighbor for node $j$ ) a Neighborhood Level $(N L)$ value is assigned. This value, $N L$, is updated (i.e., incremented or decremented) by a certain percentage according to the time it spends as a neighbor for node $j$. The Incremented Percentage (IP) is used to reflect how long two nodes stay as neighbors ( $t$ time units) and it increments the $N L$ value to reflect on this relation, on other words, the $N L$ value is updated based on the neighborhood time. Once the $N L$ reaches $100 \%$, then no increment is done.

The Decrement Percentage $(D P)$ is used to reflect how long two nodes stay as neighbors by decrementing the percentage of the neighbor node $i$ when node $i$ doesn't stay at the transmission range of node $j$. For each time $t$ when node $i$ is not found in the neighbors of node $j$, then the $D P$ will be subtracted from $N L$ of node $i$, and this scenario will be repeated for each time $t$ until the $N L$ reaches $0 \%$. At the end of this module, node $j$ will return it to all neighbors with their respective $N L$ values, which take into account the neighbors with high $N L$ values to be used in the other modules. The time complexity for this module is $O(n)$, because the calculation of the node neighbors repeated for each node in the network. And the space complexity is also $O(n)$, because each node in the network has its own table with (n) spaces.

\subsection{Initialization Module}

The second module is used to initialize the scheme main parameters: number of areas, saturation factor for area $k\left(S F_{k}\right)$, and moving exponential average $(\alpha)$ ) for node $j$ at time $t$. The module is depicted in Figure 2.

\section{Input:}

The neighbors of node $j\left(N_{j}\right)$

\section{Output:}

Number of areas

The saturation factor for areak $\left(S F_{k}\right)$

Moving exponential average $(\alpha)$ factor. 
Algorithm:

If node $j$ is first node

Then

$\mathrm{SF}_{\mathrm{k}}=0.25$

$\alpha=0.5$

Else

Exchange the information with an old node

Figure 2. Initialization Module

This module uses the output from the previous module, whereas the neighbors of node $j\left(N_{j}\right)$, to initialize the main parameters that are used in network size estimation operations.

There are several parameters that need be specified at the beginning, such as; numbers of areas (i.e. all areas have the same space). The saturation factor for area $k\left(S F_{k}\right)$ refers to the percentage of congestion in the area $k$, such that when the saturation factor is small that means slight traffic congestion in this area, on the other hand, large saturation factor values means the area is heavily congested. The summation of all saturation factors for all areas must be 1 . Other parameter is the moving exponential average $(\alpha)$ which is a constant parameter. Once a node enters a new area, it exchanges the local area information with other nodes in the area, such as: number of areas (i.e., assume grid topology with equal space for each area), the saturation factor for the area $k\left(S F_{k}=\frac{1}{A}\right)$, where $A$ is the number of areas, $\alpha=0.5$. On the other hand, when there are other nodes in area $k$, then node $j$ can use their information about the area using hello message. The output of this algorithm is that each node $j$ has its own parameters that can be used to estimate the network size. The time complexity for this module is $O(n)$, and the space complexity is constant $O(1)$.

\subsection{Estimation Module}

The Third Module estimates the number of nodes in area $k\left(N_{k}\right)$, and number of nodes in the network $(N)$ using specific operations. The module is proposed in Figure 3.

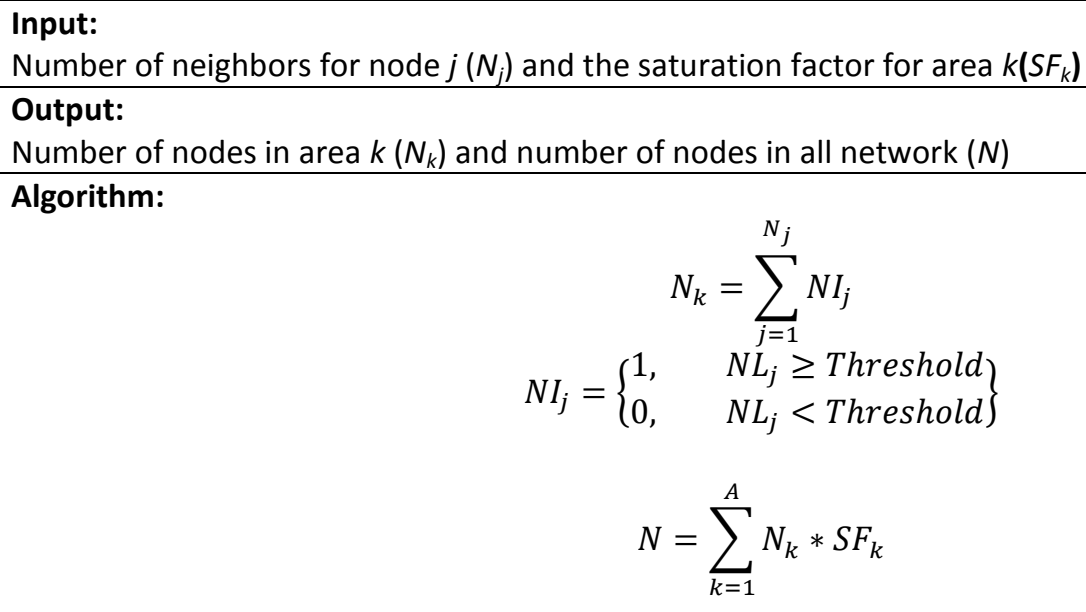

$$
\begin{gathered}
N_{k}=\sum_{j=1}^{N_{j}} N I_{j} \\
N I_{j}=\left\{\begin{array}{ll}
1, & N L_{j} \geq \text { Threshold } \\
0, & N L_{j}<\text { Threshold }
\end{array}\right\} \\
N=\sum_{k=1}^{A} N_{k} * S F_{k}
\end{gathered}
$$

Figure 3. Estimation of $\mathrm{Nk}$ and $\mathrm{N}$ Module

This module uses the number of neighbors for node $j\left(N_{j}\right)$ and the saturation factor for area $k\left(S F_{k}\right)$, which are initialized in the previous module, to estimate the number of nodes in the area that node $j$ located in, and therefore estimate the number of nodes in the network. The number of neighbors for node $j\left(N_{j}\right)$ taken from node $j$ table, that contain all neighbors 
and their $N L$ values. Only nodes with high $N L$ value (greater then certain threshold) are considered neighboring nodes for node $j$. The Saturation Factor $\left(S F_{k}\right)$ is taken from the neighbors of node $j$ (i.e., if found, by hello message), on the other hand, if no node is found in area $k$ to communicate with, then node $j$ can calculate the saturation factor using the fraction of one by the number of areas (i.e. 1/ number of area). The time complexity for this module is $O(n)$, and the space complexity is constant $O(1)$.

\subsection{Update the SFk Module}

The Fourth Module estimates the recent saturation factor and updates the saturation factor that belongs to node $j$ itself depending on the recent saturation factor. The module is proposed in Figure 4.

$\begin{aligned} & \text { Input: } \\ & \text { Number of nodes in area } k\left(N_{k}\right) \text {, number of nodes in all network }(N) \text { and moving exponential average }(\alpha)\end{aligned}$
$\begin{aligned} & \text { Output: } \\ & \text { The saturation factor for area } k\left(S F_{k}\right)\end{aligned}$
\[ \begin{array}{c}\text { Algorithm: } \\ \qquad F_{k}=\alpha * \operatorname{RecentS} F_{k}=\frac{N_{k}}{N}\end{array} \]

Figure 4. Update the SFk Module

In the third module, node $j$ use saturation factor from neighbor nodes or using fraction one by number of areas in network to guarantee the real distribution of nodes in the network. This module performs some operations to find the saturation factor for node $j$ itself. Number of nodes in area $k\left(N_{k}\right)$, number of nodes in all network $(N)$ and moving exponential average $(\alpha)$ are used as input to this module. In first operation, recent saturation factor of area $k$ is found using number of nodes in area $k$ and number of nodes in the network. RecentSF $F_{k}=$ $N_{k} / N$ such that the recent saturation factor is equal to the fraction of number of nodes in area $k$ by number of node in the network, the need of recent saturation factor come from the dynamic moving of nodes in the network which is give us dynamic distribution of nodes while moving.

In the second operation, the saturation factor of node $j$ itself is calculated using moving exponential average $(\alpha)$ and the recent saturation factor that is found in the first operation. $S F_{k}=\alpha *$ Recent $S F_{k}+(1-\alpha) * S F_{k}$ such that the saturation factor of node $j$ is equal to moving exponential average multiplied with recent saturation factor added to the result of multiplication of one minus moving exponential average with old saturation factor that node $j$ have from neighbors or by dividing one by number of areas in network. This update of saturation factor during time and moving of nodes give us closely related to real spread of nodes in the network because we work on the dynamic node and dynamic distribution. As the output of this algorithm each node $j$ has its own information about the area saturation factor that belongs to node $j$ itself and other nodes can take this information by hello message and network communication. The time complexity for this module is $O(n)$, because the calculation in each node repeated for each node in the network. And the space complexity is constant $O(1)$.

\subsection{Proposed Scheme Summary}

Figure 5depicts the flow of operations and data in the proposed scheme as well as the relationships between the 4 modules. Firstly, the node does neighbors estimation to 


\section{I Macrothink}

calculate the number of neighbors to use it in the next steps. At the second step, the nodes collect and initialize the information from neighbor nodes (e.g. the saturation factor for the area which node arrives to), then the node broadcast the hello message to find the number of neighbors. In the next step, the node estimate the $N_{k}$ (number of nodes in the area $k$ ), and use the $N_{k}$ for estimating the $N$ (number of nodes in all network). Finally, the node performs essential calculations, using the estimated $N_{k}$ and $N$, to update $S F_{k}$.
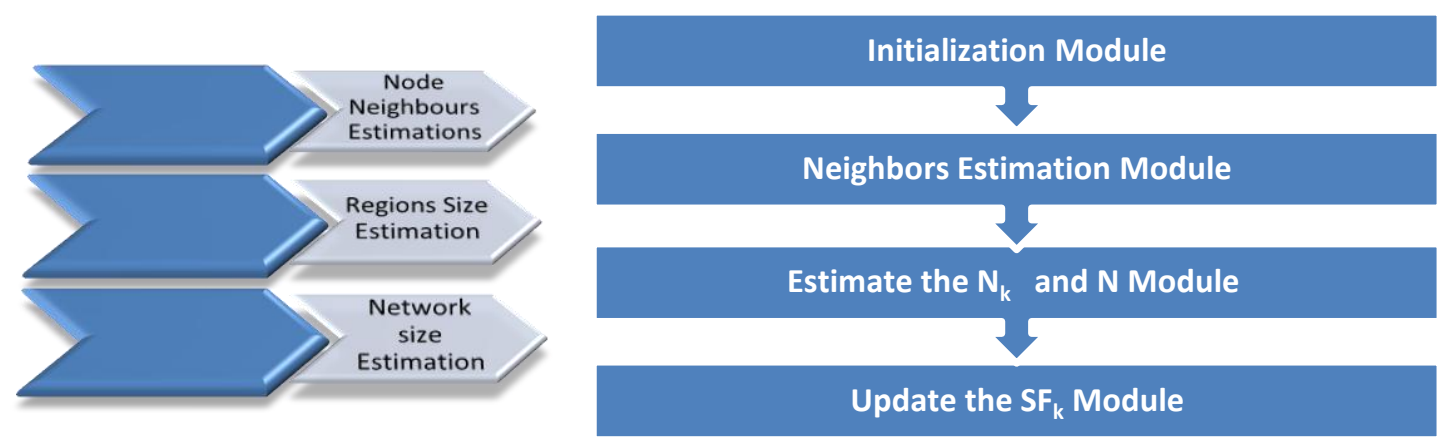

Figure 5. Proposed Scheme

\section{Simulation and Results}

To evaluate the performance of the proposed scheme, a simulation study was conducted. The algorithm is tested for various scenarios with different number of nodes and different number of areas, such that, each area represent one intersection of roads and all areas have the same size in meters. The simulation is conducted using QualNet Simulator v5.2 [40]. The most important factor in VANETs is the mobility and movement of nodes, that characterized by direction, speed and drive in reserved roads. Qualnet supports three types of mobility models which are suitable for VANETs. In the experiments, we build the configuration files for the tested scenarios. In which the vehicles mobility were described, where nodes moves in specific roads. The routing protocol is AODV. Each node is specified with node identifier and located with three directions ( $x, y$ and $z$ ). At first, all nodes are fixed in their positions, and when simulation runs the nodes move in restricted roads with variety of speeds.

\subsection{Simulation Parameters}

The run time for each experiment is 6000 seconds. And the simulation is tested with three different area sizes and numbers of nodes, with different nodes density. We divided the network into areas, such that, each area extend to $300 \mathrm{~m}$ X $300 \mathrm{~m}$ and each area covers one intersection of roads. The tested scenarios varied in number of areas; 4, 9 and 16 areas, and each scenario is tested under different densities (e.g. for scenario of 9 areas we tested 10, 25, 50,75 and 100 nodes). We generated a node placement and movement file for each scenario where every node position is specified and all movements are predefined in the reserved roads.

Furthermore, the impact of nodes speed (e.g. slow, medium and fast) is investigated. Table 1 presents the simulation parameters for the variable density scenarios. Table 2 presents the simulation parameters for the variable speed scenario. 
Table 1: Density Scenario Properties

\begin{tabular}{|l|l|}
\hline \multicolumn{1}{|c|}{ Parameters Name } & \multicolumn{1}{c|}{ Values } \\
\hline QualNet Version & 5.2 \\
\hline Simulation Time & $\mathbf{6 0 0 0} \mathrm{sec}$ \\
\hline Scenario Dimension $(x, y)$ & $(1000,1000),(1500,1500),(2000,2000)$ \\
\hline Number of intersections & 4,9 and 16 \\
\hline Distance Unit & Meter \\
\hline Number of Nodes & $10,25,50,75$ and 100 nodes \\
\hline
\end{tabular}

Table 2: Speed Scenario Properties

\begin{tabular}{|l|l|}
\hline \multicolumn{1}{|c|}{ Parameters Name } & \multicolumn{1}{c|}{ Values } \\
\hline QualNet Version & 5.2 \\
\hline Simulation Time & $\mathbf{2 0 0 0 , 4 0 0 0}$ and $6000 \mathrm{sec}$ \\
\hline Scenario Dimension $(x, y)$ & $(1000,1000),(1500,1500),(2000,2000)$ \\
\hline Number of intersections & 4,9 and 16 \\
\hline Distance Unit & Meter \\
\hline Number of Nodes & 10,25 and 50 nodes \\
\hline
\end{tabular}

For the purpose of evaluating the proposed algorithm, we used the percentage of correct estimation as a performance metric. Such that, a high percentage indicates that the proposed algorithm is able to achieve the intended goal. Figure 6 shows the scenario of 16 intersections where the red flag display the roads that restricted for vehicle moving, and each intersection of two roads represents one region. The green arrow represents CBR (constant bit rate) application.

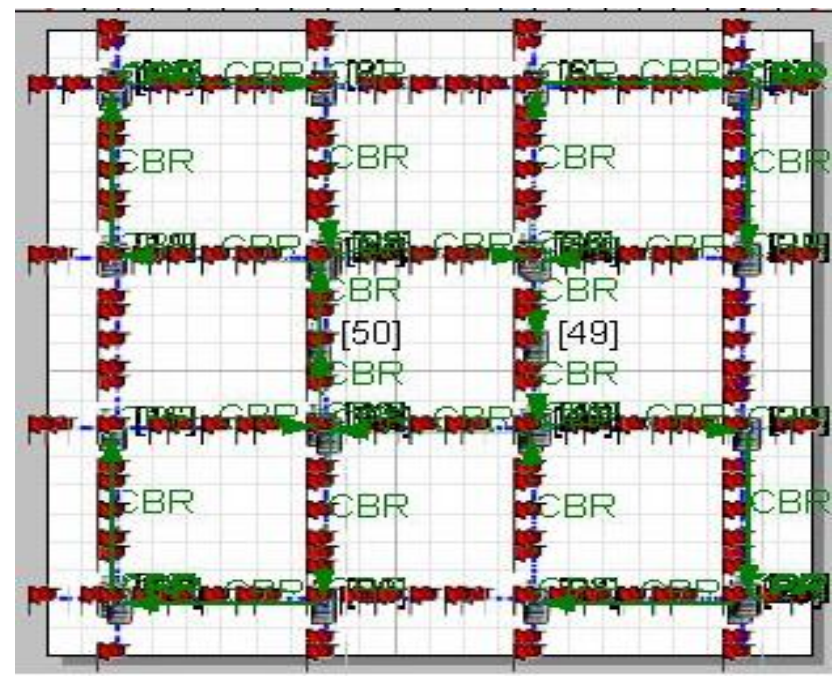

Figure 6. Network Scenario of 16 intersections

\subsection{Simulation Results}

To evaluate the performance of the proposed scheme, a number of simulation experiments were conducted. The experiments are divided into two sets. The first set of 
results represents the percentage of estimation when testing the varying density scenarios (when the number of nodes is increased). The second set of simulation results shows the impact of nodes speed on the proposed algorithm.

\subsubsection{Percentage of estimating for various densities}

Here we show the result of testing the impact of density on the proposed algorithm. At first we compare between 10 nodes and 25 nodes in 4 intersections. The number of areas in this scenario is 4, and the nodes are distributed in reserved roads in all areas. As Figure 7 shows, when the density increases (e.g. from 10 nodes to 25 nodes) the percentage of estimation decreases.

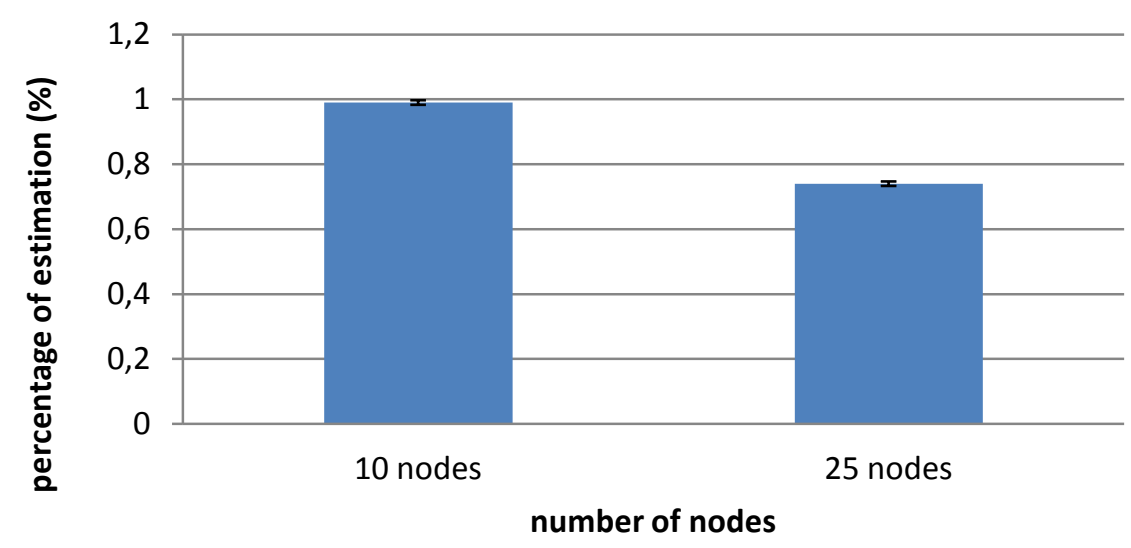

Figure 7. Network estimation for 4 intersections

For the scenario of 9 areas and number of nodes varies from 10 to 100 nodes, Figure 8 shows that when the density increases (e.g. from 10, 25, 50, 75 and 100 nodes) the percentage of estimation decreases.

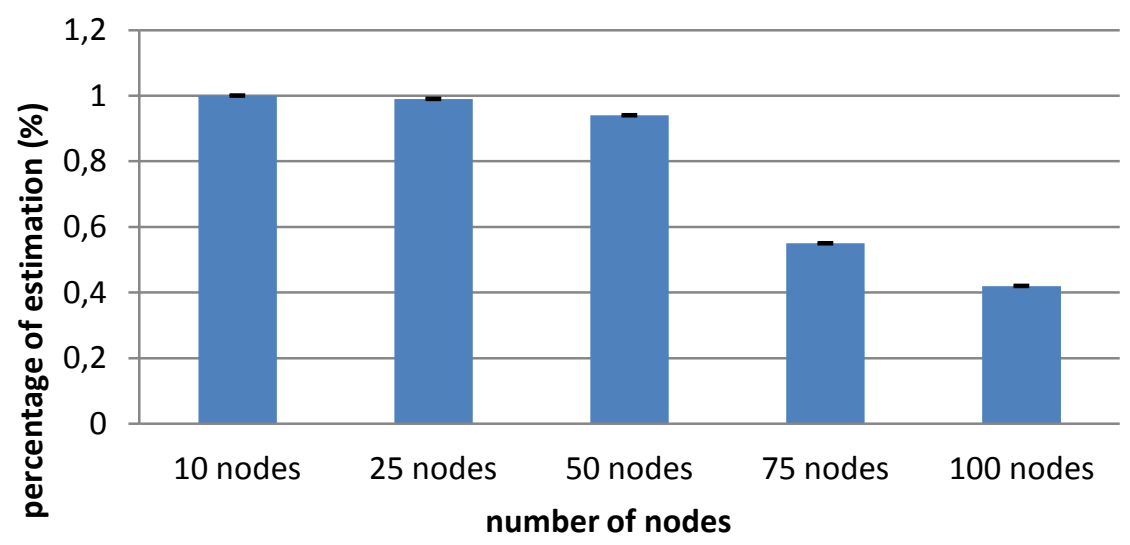

Figure 8. Network Estimation for 9 intersections

Moreover, we compare between 25, 50, 75 and 100 nodes in 16 intersections. The number of areas in this scenario is 16 areas, and the nodes are distributed in reserved roads in all areas. As figure 9 shows, when the density increases (e.g. from 25, 50, 75 and 100 nodes) the percentage of estimation decrease. 


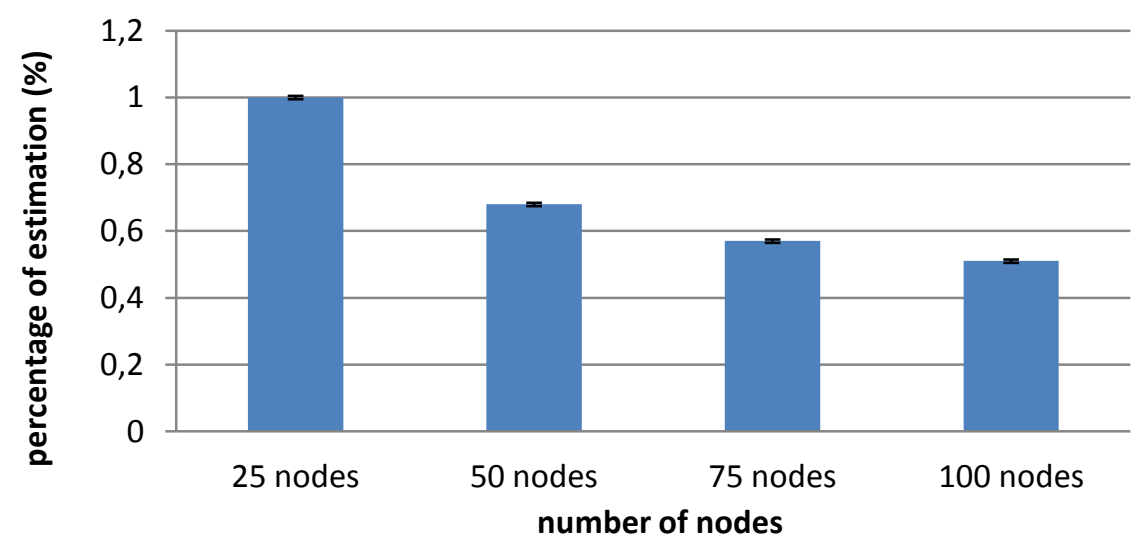

Figure 9. Network Estimation for 16 intersections

Then we perform different comparisons between the number of areas, such that; 4, 9 and 16 intersections for same number of nodes ( 25 nodes). As Figure 10 show, when the number of areas increase (same number of nodes distributed in 4 areas which give us more density, then 9 areas which give us less density, and then 16 areas which give us continue decreasing of density) the density will be decrease to achieve the needed density for estimation:

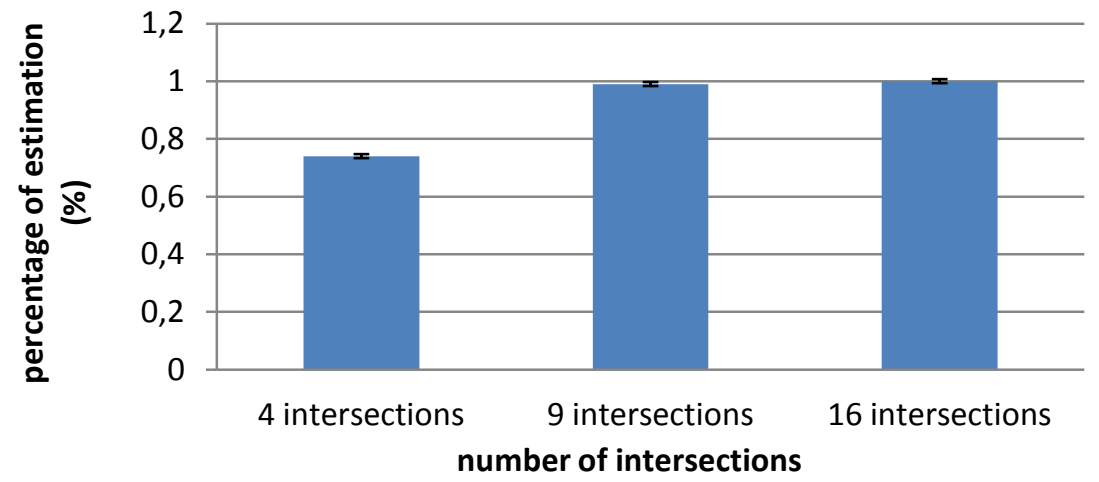

Figure 10. Network Estimation for 25 nodes

Furthermore, the effect of number of area is tested by varying number of intersections from 9 to 16 intersections using the same number of nodes (50 nodes). As Figure 11 shows, when the number of areas increases (same number of nodes distributed in 9 areas which is give us dense network and then 16 areas which is give us sparse network) the density decreases, therefore, increasing the estimation percentage.

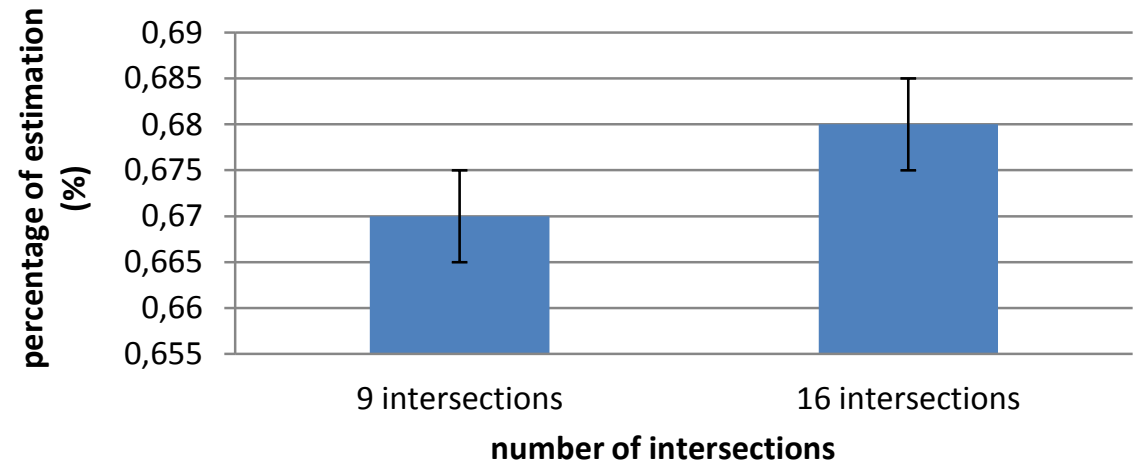

Figure 11. Network Estimation for 50 nodes 
Finally we evaluated all scenarios; 4, 9 and 16 intersections with varying number of nodes (e.g. 10, 25, 50, 75 and 100 nodes). We computed the confidence interval (95\%) for all experiments, the obtained confidence interval are very narrow. As Figure 12 shows, when the density increases the percentage of estimation decreases in some cases (e.g. 10, 25, 50, 75 and 100 nodes in 4 intersections) and in the other cases, when the network is more sparse than needed, the percentage of estimation decreases (e.g. 10 nodes in 16 intersections), this behavior is due to the small number of nodes in each area which fails to provide sufficient information for the algorithm and therefore is unable to provide an accurate estimations.

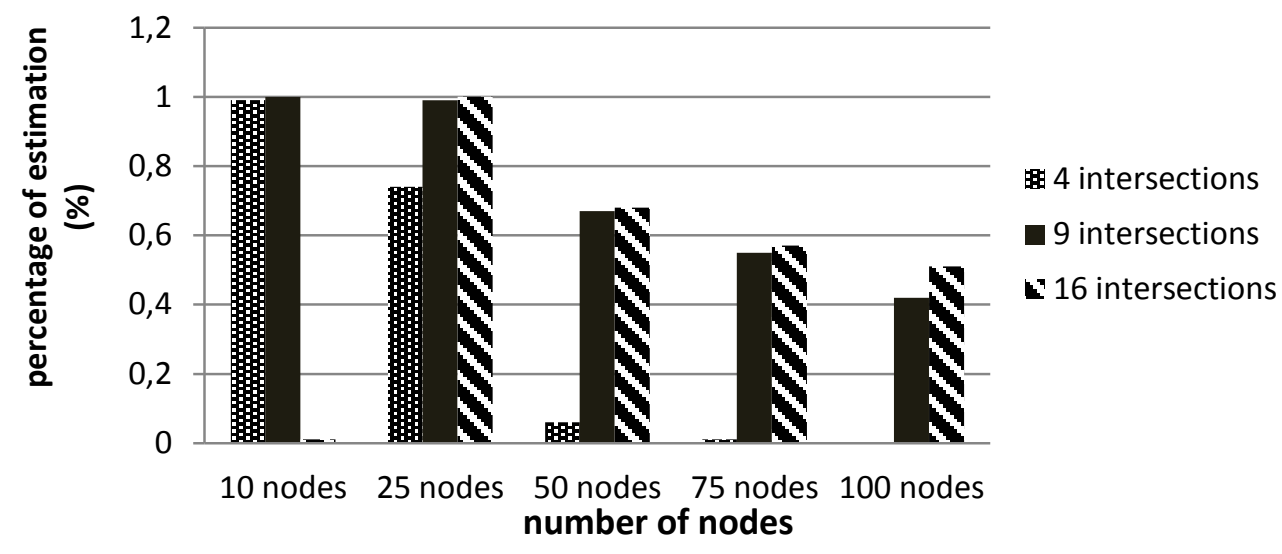

Figure 12. Network Estimation for all scenarios

\subsubsection{Percentage of estimation for various speeds}

Here we show the result of testing the impact of speed on the proposed algorithm. In Figure 13, we compare the impact of speeds of nodes on the same scenario (10 nodes and 4 intersections), when nodes moves in fast, medium and slow speeds. We computed the confidence interval $(95 \%)$ for scenario of 10 nodes and 4 intersections for all speeds, the obtained confidence interval are very narrow. The result shows that when the speed of nodes is increased the percentage of estimation is decreased. We note that the estimation depends on the information that is collected from neighbor nodes, and when the speeds of nodes are increased the information that collected is decreased.

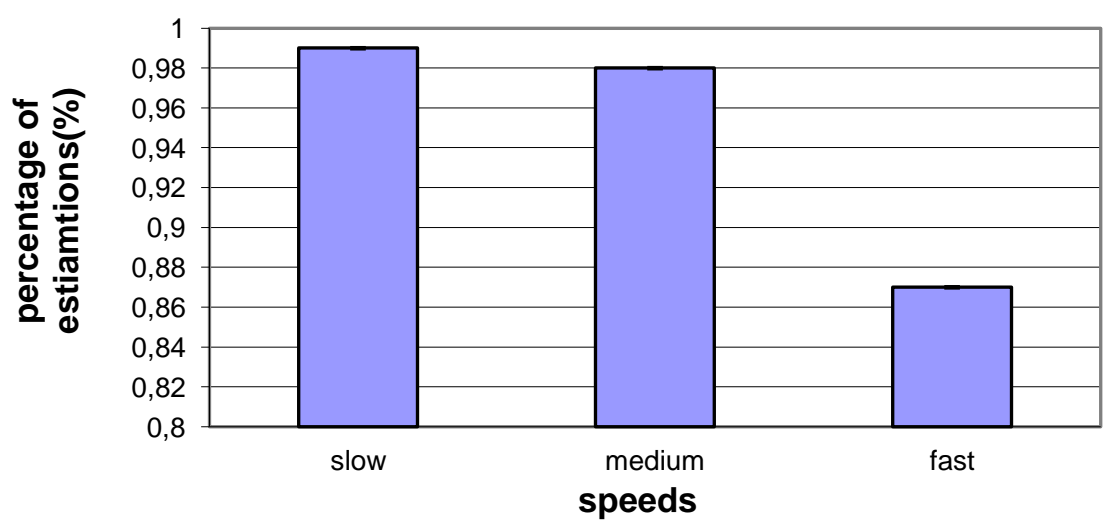

Figure 13. Network Estimation for varies mobility scenarios (10 nodes and 4 intersections)

Also, we compare the impact of speeds of nodes on the same scenario ( 25 nodes and 9 intersections), when it is moves in fast, medium and slow speeds, the results are depicted in Figure 14. We computed the confidence interval (95\%) for scenario of 25 nodes and 9 


\section{Macrothink}

intersections for all speeds, the obtained confidence interval are very narrow. The result shows that when the speed of nodes is increased the percentage of estimation decreases.

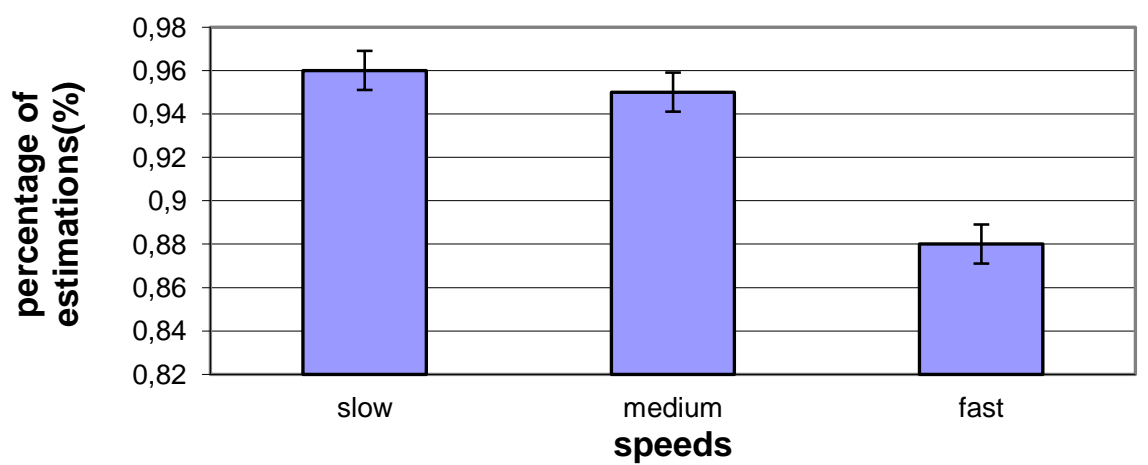

Figure 14. Network Estimation for varies mobility scenario (25 nodes with 9 intersections)

Finally, we compare the impact of nodes' speed on the same scenario (50 nodes and 16 intersections), when the nodes moves in fast, medium and slow speeds, the results of this experiments are depicted in Figure 15. We computed the confidence interval (95\%) for scenario of 50 nodes and 16 intersections for all speeds, the obtained confidence interval are very narrow

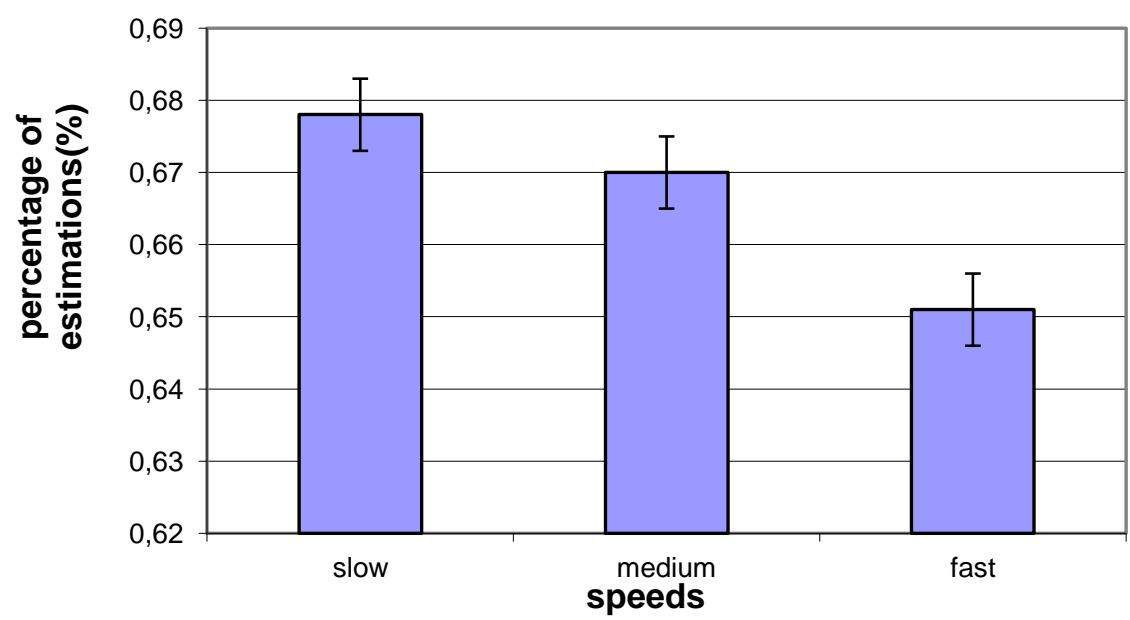

Figure 15. Network Estimation for varies mobility scenario (50 nodes_16 intersections)

The proposed scheme collects essential estimation parameters from the neighbor nodes, and when the speeds of nodes increases, the collected information decreases, therefore, it is expected that the estimation accuracy decreases as the nodes' mobility increases. Results presented in Figure 15 confirms to this conclusion. Figure 15 shows the percentage of estimation for various nodes' speeds (slow, medium, and fast). The estimation accuracy drops from $67.9 \%$ for slow network to $65.1 \%$ for the fast network.

\section{Conclusions and Future Work}

In this paper, we investigated various issues regarding network size for VANETs to produce an accurate algorithm for estimating the network size with minimal traffic overhead. 


\section{MInstitute Macrink $_{\text {Int }}$}

The best percentage of estimation was achieved when the network density is in between sparse and dense, such that, the estimation percentage for 25 nodes in 9 and 16 intersections is similar but the percentage of estimating for 10 nodes in 16 intersections is different because 10 nodes in 16 intersections produce more sparse than needed to perform the estimation. Regarding the impact of nodes moving speed, results indicates that increasing the nodes' speed results in less accurate estimations, because the algorithm depends heavily on collecting the estimation information from the neighboring nodes, which in turn are not stable with high mobility.

This paper proposed a solution to the challenging problem of network size estimation in VANETs. However, there are several research points that can be further investigated in order to extend the approach used in this paper: For future work, it would be worthwhile testing the proposed scheme under other scenarios and then adaptively tune the estimation parameters that obtain the best estimation results.

\section{References}

[1] Amit Kumar Saha and David B. Johnson, "Modeling Mobility for Vehicular Ad Hoc Networks", in Proc. 1st ACM VANET, Philadelphia, PA, New York, USA, Pages 91-92, Oct. 2004. http://dx.doi.org/10.1145/1023875.1023892

[2] K. Sampigethaya, Mi. Li, L. Huang, and R. Poovendran, "AMOEBA: robust location privacy scheme for VANET", IEEE Journal on Selected Areas in Communications (JSAC), Special issue on Vehicular Networks, Washington, USA, Volume 25, No. 8, Pages 1569-1589, Oct. 2007. http://dx.doi.org/10.1109/JSAC.2007.071007

[3] Jung-Hun Kim, Su Kyoung Lee, "Reliable Routing Protocol for Vehicular Ad Hoc Networks", AEU - International Journal of Electronics and Communications, Seoul, Korea, Volume 65, Issue 3, Pp. 268-271, March 2011. http://dx.doi.org/10.1016/j.aeue.2010.02.014

[4] Sven Jaap, Marc Bechler, and Lars Wolf, "Evaluation of Routing Protocols for Vehicular Ad Hoc Networks in Typical Road Traffic Scenarios", in Proc. of the 11th EUNICE Open European Summer School on Networked Applications, Colmenarejo, Spain, July 2005.

[5] Atulya Mahajan, "Urban Mobility Models for VANETs", IEEE Workshop on Next Generation Wireless Networks (WoNGeN), Bangalore, India, December 2006.

[6] K. C. Lee, U. Lee, and M. Gerla, "Survey of Routing Protocols in Vehicular Ad Hoc Networks", in Advances in Vehicular Ad-Hoc Networks: Developments and Challenges, M. Watfa, Ed.: Information Science Reference (IGI Global), UCLA, USA, Pages 149-170, 2010.

[7] Mohammad M. Abdellatif, "A Brief Summary on the Main Aspects and Challenges of Vehicular Ad-Hoc Networks (VANETs)", 1st MAP-Tele Doctoral Students Workshop 2010 (MAPshop'10), Porto, Portugal, 24th of February 2010.

[8] B. Liu, B. Khorashadi, D. Ghosal, C.-N. Chuah, and M. Zhang, "Assessing the VANET's Local Information Storage Capability Under Different Traffic Mobility”, in Proc. of IEEE INFOCOM 2010, San Diego, CA, USA, 14-19 March 2010. http://dx.doi.org/10.1109/INFCOM.2010.5462262

[9] Tim Leinmuller and Elmar Schoch, "Greedy Routing in Highway Scenarios: The Impact of Position Faking Nodes", In Proceedings of Workshop on Intelligent Transportation Systems (WIT 2006), German, 2006.

[10] Dinesh Kumar, Arzad A. Kherani, and Eitan Altman, "Route Lifetime Based Optimal Hop Selection in VANETs on Highway: An Analytical Viewpoint", in Proc. Netw., France, Volume 3976, Pages 799-814, 2006. http://dx.doi.org/10.1007/11753810_67

[11] Pratap Kumar Sahu, Eric Hsiao-Kuang Wu, Jagruti Sahoo, Mario Gerla, "DDOR: 
Destination Discovery Oriented Routing in Highway/Freeway VANETs", Telecommunication Systems, August 2012, Volume 50, Issue 4, pp 267-284. http://dx.doi.org/10.1007/s11235010-9403-2

[12] M. Jerbi, S. Senouci, R. Meraihi, Y. Ghamri-Doudane, "An Improved Vehicular Ad Hoc Routing Protocol for City Environments", IEEE International Conference on Communications (ICC 2007), Glasgow, Scotland, UK, June 2007. http://dx.doi.org/10.1109/ICC.2007.654

[13] Bruce Wang, Teresa M. Adams, Wenlong Jin, Qiang Meng, "The Process of Information Propagation in a Traffic Stream with a General Vehicle Headway: A Revisit", Transportation Research Part C: Emerging Technologies, USA, Volume 18, Issue 3, Pages 367-375, June 2010. http://dx.doi.org/10.1016/j.trc.2009.05.011

[14] Marco Fiore, Jerome H"arri, FethiFilali, Christian Bonnet, "Vehicular Mobility Simulation for VANETs", IEEE International Annual Simulation Symposium (ANSS), Norfolk, VA, USA, March 2007. http://dx.doi.org/10.1109/ANSS.2007.44

[15] FelizKristianto Karnadi, "Rapid Generation of Realistic Mobility Models for VANET", Wireless Communications and Networking Conference, WCNC, Australia, 2007. http://dx.doi.org/10.1109/WCNC.2007.467

[16] M. Gerla, X. Hong, and G. Pei, "Fisheye State Routing Protocol (FSR) for Ad Hoc Networks", IETF Internet Draft, July 2002.

[17] C. Perkins, E. Royer, and S. Das, "Ad hoc On-Demand Distance Vector (AODV) Routing", RFC 3561, USA, July 2003.

[18] V. Park and S. Corson, "Temporally-Ordered Routing Algorithm (TORA) Version 1: Functional Specification", IETF Internet Draft, July 2001.

[19] D. Johnson, D. A. Maltz, and Y.-C. Hu, "The Dynamic Source Routing Protocol for mobile Ad hoc Networks (DSR)", IETF Internet Draft, July 2004.

[20] B. Karp, H.T. Kung, "Gpsr: Greedy Perimeter Stateless Routing for Wireless Networks", 6th International Conference on Mobile Computing and Networking, Boston, MA, USA, Pages 243-254, 2000.

[21] C. Lochert, H. Hartenstein, J. Tian, H. F"ussler, D. Hermann, and M. Mauve, "A Routing Strategy for Vehicular Ad hoc Networks in City Environments", in Proc. IEEE IV'03, Germany, Pages 156-161, Jun 2003.

[22] David Oliver Jorg; "Performance Comparison of MANET Routing Protocols in Different Network Sizes", Institute of Computer Science and Applied Mathematics, Computer Networks and Distributed Systems (RVS), University of Berne, Switzerland, 2003.

[23] A. Boomarani Malany, V.R. Sarma Dhulipala, and RM. Chandrasekaran "Throughput and Delay Comparison of MANET Routing Protocols", Int. J. Open Problems Compt. Math., India, Volume 2, No. 3, September 2009.

[24] Prabhakar Ranjan and Kamal Kant Ahirwar " Comparative Study of VANET and MANET Routing Protocols", International Conference on Advanced Computing and Communication Technologies (ACCT 2011), 20-22 January 2011. Gymkhana Club, HUDA Sector 4, Rohtak (INDIA).

[25] T. Taleb, E. Sakhaee, A. Jamalipour, K. Hashimoto, N. Kato and Y. Nemoto, "A Stable Routing Protocol to Support ITS Services in VANET Networks", IEEE Transactions on Vehicular Technology, Australia, Volume 56, No 6, 2007. http://dx.doi.org/10.1109/TVT.2007.906873

[26] O. Tonguz, N. Wisitpongphan, F. Bai, P. Mudalige, and V. Sadekar, "Broadcasting in VANET", in INFOCOM MOVE Workshop 2007, Anchorage, Alaska, USA, 11 May 2007. http://dx.doi.org/10.1109/MOVE.2007.4300825

[27] C. Sommer and F. Dressler, "The DYMO Routing Protocol in VANET Scenarios", In 66th IEEE Vehicular Technology Conference (VTC2007-Fall), pages 16-20, Baltimore, 
Maryland, USA, September/October 2007. http://dx.doi.org/10.1109/VETECF.2007.20

[28] R. Baumann, "Vehicular Ad hoc Networks (VANET) - Engineering and Simulation of Mobile Ad hoc Routing Protocols for VANET on Highways and in Cities", Master's Thesis in Computer Science, ETH Zurich, 2004.

[29] A. Boukerche, H. A. B. F. Oliveira, E. F. Nakamura, and A. A. F. Loureiro, "Vehicular Ad Hoc Networks: A New Challenge for Localization-Based Systems", Computer Communications, Canada, Volume 31, Pages 2838-2849, 2008. http://dx.doi.org/10.1016/j.comcom.2007.12.004

[30] T. Kosch, C. J. Adler, S. Eichler, C. Schroth, and M. Strassberger. "The Scalability Problem of Vehicular Ad hoc Networks and How to Solveit", IEEE Wireless Communications, Volume 13, Pages 22-28, October 2006.

[31] J. Nzouonta, N. Rajgure, Guiling Wang, C. Borcea, "VANET Routing on City Roads Using Real-Time Vehicular Traffic Information", IEEE Transactions on Vehicular Technology, USA, Volume 58, no.7, Pages 3609-3626, Sept. 2009. http://dx.doi.org/10.1109/TVT.2009.2014455

[32] S. Raghay, S. El Hadaj and T. Noel3, "A Genetic Algorithm for Management Data Stream in VANET", International Journal of Information Technology and Knowledge Management, Volume 4, No. 1, Pages 315-318, January-June 2011.

[33] Bijan Paul, Md. Ibrahim, Md. Abu Naser Bikas, "Performance Evaluation of AODV and DSR with Varying Pause Time and Node Density Over TCP and CBR Connections in VANET", International Journal of Computer Science and Network Security (IJCSNS), Bangladesh, Volume 11, Issue 7, July 2011.

[34] Rahat Ali Khan, Shakeel Ahmed Shah, Muhammad Abdul Aleem, Zulfiqar Ali Bhutto, Asad Ali Shaikh and Muhmaad Aslam Kumbhar, "Wireless Sensor Networks: A Solution for Smart Transportation", Journal of Emerging Trends in Computing and Information Sciences, Pakistan, Volume 3, No. 4, April 2012.

[35] Marco Gramaglia, "A Reliable Multicast Routing Approach for VANET", IJCSI International Journal of Computer Science Issues, Volume 9, Issue 2, No 2, March 2012.

[36] R.K. Chauhan and Arzoo Dahiya, "AODV Extension Using Ant Colony Optimization for Scalable Routing in VANETs ", Journal of Emerging Trends in Computing and Information Sciences, Kurukshetra, Volume 3, No. 2, February 2012.

[37] Uma Nagaraj and Poonam Dhamal, "Broadcasting Routing Protocols in VANET", Network and Complex Systems, Volume 1, No.2, 2011.

[38] H. Meyer, O. Trullols, A. Hess, K. Hummel, J. Barcelo, C. Casetti, and G. Karlsson, "VANET Mobility Modeling Challenged by Feedback Loops," in 10th IEEE/IFIP Annual Mediterranean Ad Hoc Networking Workshop (IEEE/IFIP Med-Hoc-Net 2011), Favignana Island, Sicily, 12-15 June 2011. http://dx.doi.org/10.1109/Med-Hoc-Net.2011.5970499

[39] T. Shafaat, A. Ghodsi, and S. Haridi, "A Practical Approach to Network Size Estimation for Structured Overlays," in Proceedings of the 3rd International Workshop on Self-Organizing Systems (IWSOS 2008), Vienna, Austria, December 10-12, 2008. http://dx.doi.org/10.1007/978-3-540-92157-8_7

[40] Scalable Network Technologies, "Qualnet simulator," Software Package, 2003. [Online]. Available: http://www.qualnet.com .

\section{Copyright Disclaimer}

Copyright reserved by the author(s).

This article is an open-access article distributed under the terms and conditions of the Creative Commons Attribution license (http://creativecommons.org/licenses/by/3.0/). 